\title{
Validation of an MRI-based method to assess patellofemoral joint contact areas in loaded knee flexion in vivo
}

\author{
Authors Names and Degrees: \\ Emily J. McWalter, $\mathrm{PhD}^{1}$ \\ Colm M O’Kane PhD, David P FitzPatrick PhD² \\ David R. Wilson, DPhil ${ }^{3}$
}

\author{
Author Affiliations: \\ ${ }^{1}$ Department of Mechanical Engineering, University of British Columbia \\ ${ }^{2}$ Department of Mechanical Engineering, University College Dublin \\ ${ }^{3}$ Department of Orthopaedics, University of British Columbia
}

\section{Corresponding Author:}

Dr. David R. Wilson

Address: 3114 - 910 West 10th Avenue, Vancouver, BC, V5Z 4E3, Canada

Phone: 604-875-4428

Fax: 604-875-4851

Email: dawilson@interchange.ubc.ca

\section{Grant Support:}

Canadian Institutes of Health Research (Operating Grant MOP-106680), Natural Sciences and Engineering Research Council, Canadian Arthritis Network, Michael Smith Foundation for Health Research, Ireland Canada University Foundation

Running Title: Patellofemoral Contact Area Validation 


\section{Validation of an MRI-based method to assess patellofemoral joint contact areas in loaded knee flexion in vivo}




\section{Abstract (max 200 words)}

Purpose: To develop and validate short axial and sagittal MRI scans $(<1 \mathrm{~min})$ to assess in vivo patellofemoral contact areas in loaded knee flexion.

Materials and Methods: Contact area was assessed in four cadaver knee specimens from axial and sagittal scans using two contact area extraction techniques ('delineation' and 'intersection') and three calculation techniques (slice thickness multiplication, linear interpolation and spline interpolation). Error was expressed as the mean absolute and percentage difference from a dye staining-based reference standard. Intra-reader and intra-subject repeatability, expressed as the mean standard deviation, was determined.

Results: Contact area assessments from the sagittal MRI scans using the delineation and slice thickness multiplication technique had the smallest error $\left(47.7 \pm 38.1 \mathrm{~mm}^{2}\right.$ or $\left.10.7 \%\right)$. The intra-reader repeatability from assessments using the sagittal scans was smaller than those using the axial scans when the delineation method was used $\left(<9.4 \pm 4.3 \mathrm{~mm}^{2}\right.$ and $<15.4 \pm 14.1 \mathrm{~mm}^{2}$, respectively). The intra-subject repeatability of the assessment from the sagittal scan was less than $39.9 \pm 23.0 \mathrm{~mm}^{2}$.

Conclusion: This protocol yields assessments of contact area in less than 1 minute that have errors similar to those made using scans many times longer and can be used in series with kinematic scans to carry out simultaneous assessments in vivo to study patellofemoral joint disease.

Key words: Patella, Patellofemoral, Contact Area, Magnetic Resonance Imaging, Validation, Kinematics 


\section{Introduction}

Many patellofemoral disorders are thought to be related to abnormal kinematics (motion) of the patella which produce an abnormal distribution of loads in the patellofemoral joint. Measurements of patellofemoral mechanics are used to study how effectively treatments restore mechanics to normal.

5 Methods using MRI have provided measurements of three-dimensional patellar kinematics and patellar contact areas in vivo while patients load their knee without the risks, such as ionizing radiation exposure and infection, of other approaches. A primary limitation of MR-based approaches is that the scan time is sufficiently long that participants, particularly those with joint disorders, are unable to load their joints beyond a fraction of physiological levels during the scan. Assessing patellar kinematics and contact areas in loaded joints requires a validated, rapid imaging protocol.

Quantification of contact area from MRI has been carried out using both axial and sagittal scans and various different calculation methods. The length of these scans ranges from 39 seconds (1) to 11 minutes (2), with the validated techniques in the 2 to 11 minute range (2-6). Most assessments have been carried out using MRI scanners with field strengths of 1.5 Tesla or lower (1,3,5-11). It is important that

15 the knee is loaded during the contact area assessments and this has been accomplished using a vertical open configuration scanner in which the participant is in an upright position bearing $45 \%$ of their body weight through the study knee (3,5), using a horizontal open configuration scanner in which the participant is lying on his or her side resisting a $10 \mathrm{Nm}$ torsional load applied to the shank with a pulley system (6), or using traditional closed bore scanners in which the participant in a supine position and axial

20 load applied to the foot (range from $10 \%$ of maximum isometric contraction (10.5 to $13 \mathrm{~N}$ ) (4) to $25 \%$ BW $(1,12))$. In all cases, the participant must remain still for the duration of the scan, therefore the applied loads must be low enough to be maintained by the subject without substantial tremor. These applied loads are typically lower than those experienced during regular loaded activity. To assess contact area, first cartilage contact must be determined in each MRI slice; this has been carried out by delineating 
25 contact area directly $(2,3,5,8,9,12,13)$ or by defining contact as the intersection of segmented cartilage plates that have been dilated by a pixel (6). Finally, contact area must be calculated and this has been accomplished by multiplying in-slice contact by slice thickness (1-3,5,6), interpolating between slices $(8,9,13)$ or surface fitting (4). Most groups have reported only contact area, although two studies have also reported contact centroid location with respect to the patella $(4,13)$.

Limited data exist on the measurement errors associated with these methods. Errors, expressed as coefficient of variation (CV), were reported to be $3 \%$ in a phantom-based validation study (5) and $13 \%$ in a cadaver-based validation study (2), both using sagittal scans. Errors in assessments from axial scans have not been assessed. Various types of repeatability have been quantified and, when expressed as CV, in general have ranged between $3 \%$ and $12 \%(2,5,6,8,9,12)$. It is important that each method be validated

35 independently because of differences in the MRI scan sequences and calculation method used.

It is currently not possible to assess accuracy in vivo because there is no agreed-upon reference standard. Cadaver specimens provide a useful model for validating MRI based contact area measurements. They are advantageous because tissue structures and joint geometry are similar between the validation and actual in vivo measurements. Many techniques exist to assess joint contact areas in 40 cadaver specimens such as dye staining (14-17), casting (18-20), pressure sensitive film (21-25), stereophotogrammetry $(19,26)$ and dynamic pressure measurement systems (27-29). Agreement between these techniques has been shown to be very good by visual observation of registered areas (19). These techniques can be used to validate the MRI-based measures of contact area.

There currently exists no rapid, fully validated protocol for assessment of patellofemoral contact 45 areas in vivo. A scan less than one minute is required so that individuals with patellofemoral OA are able to complete the loading task without substantial tremor. With 3 Tesla MRI scanners, this shorter scan time is possible. Further, variability exists between methods of calculating contact areas from MRI in terms of the scan length and direction used, the calculation methods, and the level of validation. 
Therefore the aims of this study were: 1) To develop an axial and a sagittal MRI scan sequence of less

50 than 1 minute to be used to assess patellofemoral contact area during loaded knee flexion; 2) To develop an analysis tool that allows for calculation of contact area magnitude and location on the patellar surface;

3) To determine the error in contact area assessments from axial and sagittal MRI scans with reference to a dye staining technique in a cadaver model; 4) To determine the intra-reader and intra-subject repeatability of the technique. 


\section{Methods}

\section{Specimen Preparation}

Four cadaver knee specimens, with no history of knee surgery, paralysis, lower limb abnormalities or multiple sclerosis, were used in this study. Specimens were thawed at room temperature overnight. Soft tissues were then removed from the proximal and distal ends of the specimen, exposing approximately $12 \mathrm{~cm}$ of the femoral shaft and $8 \mathrm{~cm}$ of the tibial shaft. A longitudinal incision was made from the distal end of the patella to the proximal end of the specimen along the femoral shaft and the skin and fat layers were resected. The quadriceps tendon was dissected and the patellofemoral joint capsule was released along the proximal, medial and lateral margins of the patella. The femoral shaft was potted

$6510 \mathrm{~cm}$ from the proximal end in a custom polyvinyl chloride connector using standard dental stone. Lengths of non-stretch, ultra-high-molecular-weight polyethylene line (Spectra, Honeywell, Morristown, NJ, USA) were sutured to the quadriceps tendon and the distal end of the tibia. The fat and skin layer was then sutured closed. This was carried out in order to maintain a normal tissue layer structure for MR imaging.

\section{Experimental Procedure}

Two sagittal MRI scans (Intera, Philips, the Netherlands), one optimized for bone and the other for cartilage, of each specimen were acquired (Table I) with the specimen unloaded. The specimen was then mounted in a custom designed loading apparatus (Figure 1). The femoral end was connected rigidly to the apparatus using a nylon bolt. The line connected to the quadriceps tendon ran through a pulley

75 system and was locked down at the prescribed knee flexion angle using a plastic one-way rope anchor. The line connected to the tibia also ran through a pulley system and a $60 \mathrm{~N}$ load was applied (using MR safe sandbags) to ensure patellofemoral contact. Once loaded, three additional MRI scans were acquired (Table I): 1) sagittal low resolution TI weighted spin echo sequence, optimized for bone 2) axial T1 
weighted, three-dimensional fast field echo sequence, optimized for cartilage and 3) sagittal T1 weighted, three-dimensional fast field echo sequence, optimized for cartilage.

A dye exclusion method was used to identify regions of patellofemoral contact. To do this, the MRI bed was slowly retracted from the MRI scanner, ensuring that the loading setup was not altered. The sutures attaching the skin and fat layers were released using an MRI-safe scalpel, allowing for visualization of the loaded quadriceps tendon and the patella. Food grade dye (Blue Neon, Club House,

85 McCormick Canada, London, ON) was applied liberally to the region surrounding the patellofemoral contact. The excess dye was carefully removed using absorbent cotton pads. The load was released and the quadriceps tendon was pulled distally exposing the articular surfaces of the patella and the femur. The region of the patella devoid of dye indicated regions of patellofemoral contact.

The specimen was transported from the MRI centre to a biomechanics laboratory for assessment of contact area from the dye-based reference standard. Four fiducial markers were rigidly attached to the margins of the patella. The fiducial markers and the periphery of the contact area were digitized by tracing them using a digitizing stylus with an optical tracking system (Optotrak Certus, Northern Digital Inc, Waterloo, ON). This process was repeated 3 times. The manufacturer quoted accuracy of the system

95 is $0.15 \mathrm{~mm}$ in three dimensions. The surface topology of the patella was determined using a threedimensional non-contact digitizer (Vivid 9i, Konica-Minolta, Osaka, Japan). The manufacturer quoted accuracy of this system is $0.05 \mathrm{~mm}$ and the precision is $0.008 \mathrm{~mm}$. In practical application, one independent validation study found the agreement between the digitizer and a tactile surface scanner to be $0.08 \mathrm{~mm}$ for the reference objects and the repeatability to be $0.003 \mathrm{~mm}$ based on two scans of each of the reference objects (30). 


\section{Contact Area Analysis}

To assess contact area using the MR images, two different methods were used to identify regions of in-slice contact using commercially available image processing software (Analyze 8.0, Analyze Direct, Overland Park, KS, USA). Each method was used on both the axial and sagittal scans. In the first 105 method, 'delineation', contact (defined as no visible separation between cartilage plates) was defined directly by tracing in a slice-by-slice manner using the Image Edit module (Figure 2). The second method, 'intersection', was a multi-step process. First, the patellar and femoral trochlear cartilage was segmented independently in a slice-by-slice manner, also using the Image Edit module (Figure 2). Next, the segmented cartilage maps were converted into binary images and the femoral trochlear surface was

110 dilated by 1 pixel using the Morphology module. Finally, intersection between the patellar cartilage and dilated femoral trochlear cartilage was defined as the Boolean intersection using the Image Calculator module. Contact regions defined on the axial and sagittal images using the delineation and intersection methods were imported into custom written software (Matlab, The Mathworks, Natick, MA, USA). Splines were fit to the in-slice contact data. Area was then calculated in three different ways: 1) by

115 finding the sum of the products of each in-plane spline and the slice thickness; 2) by carrying out a linear interpolation between splines in adjacent slices and summing the area of the triangle faces created by the interpolation; 3) by fitting splines between slices and summing the area of the triangle faces created by the interpolation. The number of points sampled along the in-plane and between-plane splines was determined in a case specific manner and was defined as the number of points required for the area calculation to change by less than $0.01 \mathrm{~mm}^{2}$.

The assessment of contact area from the dye-based reference standard was carried out using custom written software (Matlab, The Mathworks, Natick, MA, USA). The digitized contact area was registered to the patellar surface using the surface topology data, the fiducial marker locations and an Iterative Closest Points (ICP) algorithm. The registered data were imported into commercial laser 
125 scanning/solid modelling software (Rapidform XOR, INUS Technology Inc., Seoul, South Korea) and a surface mesh was created. This process included filling holes, finding defects in the mesh, smoothing the outer boundary and optimizing the mesh, all using tools built into the commercial software. This type of processing is standard for laser scanner data (30). A non-uniform, rational, basis spline (NURBS) surface was fit to the optimized mesh; the difference between the mesh and the NURBS surface was never more

130 than $0.1 \mathrm{~mm}$. A spline was fit to the dye contour, which was projected onto an oblique plane containing the four posterior points on the NURBS surface. The projected profile of the dye contour was extruded through the NURBS surface, which was then used to cut the extrusion. The result was a NURBS surface describing the contact area. Area of the contact surface was calculated using a built in tool. This process was carried out for all 3 sets of dye-based contours.

135 In order to determine the position of the MRI-based contact areas relative to the reference standard, the data were plotted in the anatomical patellar coordinate system used in the kinematic assessment method developed previously $(31,32)$. In order to accomplish this, the bone and cartilage from the unloaded MRI scans and the loaded bone scan were segmented in a slice-by-slice manner. The patellar coordinate system was defined on the unloaded bone model and therefore all data were registered

140 to this model. The unloaded patellar cartilage was already expressed in this coordinate system because the image field of view and the specimen position was the same for both scans. The contact area from the reference standard was plotted in the patellar coordinate system by registering the laser scanned cartilage surface to the unloaded MRI cartilage surface using an ICP algorithm. Plotting the MRI-based contact area in the patellar coordinate system required four steps: 1) the bone was segmented from the loaded 145 bone scan in a slice-by-slice manner and contours were created; 2) the loaded bone contour and the loaded contact area were transformed into the same coordinate system using a homogenous transformation matrix created using the Image Patient Position (coordinate of upper left hand corner of the image) and Image Orientation Patient (direction cosines) tags from the DICOM images; 3) the bone contour was registered to the bone model using an ICP (33) and the homogeneous transformation matrix 
was calculated; 4) the contact area was transformed using the homogeneous transformation matrix from step 3. The MRI-based and reference standard contact areas were plotted in the patellar coordinate system and contact centroids were calculated for each (1 MRI and 3 trials of reference standard). The distance between the MRI-based contact centroid and the three reference standard contact centroids was calculated. The percentage overlap between the MRI and reference standard was also determined using 155 the solid modelling software.

\section{Accuracy}

The error of the MRI based assessment of contact area as compared to the reference standard was defined as the mean absolute and percent difference between MRI-based assessment and the mean of the three reference standard assessments. The distance between the MRI and reference standard centroids and the mean percentage overlap of the MRI and reference standard areas were also calculated.

\section{Repeatability}

The intra-reader repeatability of the axial and sagittal MRI-based assessment of contact area was determined by repeating the delineation and intersection analysis 3 times for all datasets. Intra-reader repeatability was expressed as the mean standard deviation of the assessment.

Intra-subject repeatability of the MRI-based measure of contact area was determined in two normal healthy subjects. The study protocol was approved by the institutional review board and informed consent was obtained from each participant. Unloaded bone and cartilage scans were acquired with the knee coil (Table I). Loaded sagittal bone and cartilage scans were acquired at six different angles of knee flexion between full extension and approximately $45^{\circ}$. The knee was loaded to $15 \% \mathrm{BW}$ using a custom designed loading device (34). The loaded bone and cartilage scans were acquired in series without a break and therefore the total loading time was 1 minute 35 seconds for each flexion angle. Two additional sets of 6 loaded scans were acquired with a 10 minute break between sets during which the 
participant left the scanner. Only sagittal cartilage images were acquired because they showed better agreement with the reference standard and had lower intra-reader repeatability (see results section).

175 Contact area was assessed at each angle of knee flexion using the delineation method described above. Contact area was plotted as a function of knee flexion and splines were fit to each repeated set. Repeatability was expressed as the mean standard deviation of contact area sampled at $1^{\circ}$ increments along the fitted spline for the coincidental range of knee flexion. This study was approved by the institutional review board and informed consent was obtained from each participant. 


\section{Results}

For the four cadaver knee specimens (3 right, 1 left, 2 female, mean age 70.0 \pm 5.5 years), the sagittal MRI scans consistently showed better agreement with the reference area than the axial scans; mean error for the three calculation types ranged from 47.7 to $68.4 \mathrm{~mm}^{2}$ for the sagittal scans and from 61.4 to $175.6 \mathrm{~mm}^{2}$ for the axial scans (Table II). For the sagittal scans, the delineation method had less error than the intersection method; errors ranged from 47.4 to $64.1 \mathrm{~mm}^{2}$ for delineation and from 59.4 to $68.4 \mathrm{~mm}^{2}$ for intersection (Table II). The registration error between the digitized contact area contour and the cartilage surface topology determined from the laser scan was $0.19 \pm 0.12 \mathrm{~mm}$ (expressed as the mean absolute difference between point cloud models).

The mean distance between MRI-based centroid (calculated from the delineation results from the sagittal scan) and the reference standard centroids was $2.4 \pm 1.1 \mathrm{~mm}$ (Figure 3) and the mean overlap between contact patches obtained using the two methods was $82.6 \pm 12.4 \%$. The MRI centroids were positioned $0.4 \pm 0.4 \mathrm{~mm}$ posteriorly, $0.2 \pm 0.9 \mathrm{~mm}$ medially and $1.5 \pm 2.1 \mathrm{~mm}$ proximally to the reference standard centroids in the anatomically based patellar coordinate system. The registration error of the

195 MRI-based cartilage surface model (created from the unloaded scan) to the laser scan based cartilage surface model (expressed as the mean absolute difference between models) required to assess overlap was $0.41 \pm 0.37 \mathrm{~mm}$.

The MRI-based intra-reader repeatability of the delineation method was superior to that of the intersection method (Table III); mean standard deviations ranged from 8.4 to $15.4 \mathrm{~mm}^{2}$ for the delineation method and from 20.8 to $26.2 \mathrm{~mm}^{2}$ for the intersection method. Overall, assessments from sagittal scans were more repeatable than axial; mean standard deviations ranged from 8.4 to $26.2 \mathrm{~mm}^{2}$ for the axial scans and 9.1 to $22.9 \mathrm{~mm}^{2}$ for the sagittal scans. Further, the results using the delineation method for the sagittal scans were the most repeatable between calculation methods, with mean standard deviations of 
9.4, 9.1 and $9.3 \mathrm{~mm}^{2}$ for the multiplication by slice thickness, the linear interpolation and the spline 205 interpolation methods, respectively.

The intra-subject repeatability, assessed in 2 subjects (31 year old female, 29 year old male), expressed as the mean standard deviation at $1^{\circ}$ along the spline, was $38.2 \pm 19.7 \mathrm{~mm}^{2}, 39.9 \pm 23.0 \mathrm{~mm}^{2}$, and $39.8 \pm 23.1 \mathrm{~mm}^{2}$, using the multiplication by slice thickness, the linear interpolation and the spline interpolation methods, respectively (Figure 4). Generally, contact areas increased with knee flexion

210 (Figure 5). Contact centroids translated proximally and medially with knee flexion; however, all centroids remained in the lateral compartment of the patella (lateral to the origin of the patellar coordinate system). 


\section{Discussion}

We developed short axial and sagittal MRI scans to assess articular cartilage contact areas in vivo during loaded knee flexion. The area calculations from the sagittal scans using the delineation and slice thickness multiplication technique had the lowest error $\left(47.7 \pm 38.1 \mathrm{~mm}^{2}, 10.7 \%\right)$. Intra-reader and intrasubject repeatability which were within reasonable range for this technique $\left(9.4 \pm 4.3 \mathrm{~mm}^{2}\right.$ and $38.2 \pm 19.7$ $\mathrm{mm}^{2}$ ). Contact area centroid locations were also determined to be a useful when plotted in an

220 anatomically based coordinate system. The participants could support 15\% of their body weight for the duration of the scans, which suggests that these protocols can be used to determine contact area for nearphysiological loads; this load is similar to the swing phase of gait (35). Sagittal scans and the delineation and slice thickness multiplication method should be used to assess patellofemoral contact areas in vivo during loaded knee flexion using MRI.

Our contact area errors for sagittal scans were similar to results reported in the literature, but our scan time was substantially shorter. A cadaver-based validation study that reported an average percentage difference $10.9 \%$ and a CV of $13 \%$ used a sagittal scan and a delineation and multiplication by slice thickness technique to calculate contact area (2). Our error of agreement with the reference standard had quite a large standard deviation, which is consistent with the previous cadaver validation study 230 (differences of up to $25 \%$ with the pressure sensitive film-based reference standard (2)). While those results were similar to those of the present study using a similar technique (10.7\%), the scan sequence used in that study was 11 minutes. A validation study that utilized a contact phantom and a scan sequence of 2 minutes 13 seconds had superior agreement with the reference $(\mathrm{CV}=3 \%)$ than ours; however, this is likely due to the symmetry of the phantom (circular contact) and the high signal contrast between the 235 contact and surrounding material in the phantom (the boundary was clearly discernable). Our intra-reader repeatability (less than $9.4 \mathrm{~mm}^{2}$ for the sagittal scans) is in the range of what has been reported previously (1.3 $\mathrm{mm}^{2}$ with an axial scan 39 seconds in length (12) and $21 \mathrm{~mm}^{2}$ with an axial scan 6 minutes and 30 
seconds in length (9)). Our intra-subject repeatability (10.4-11.3\%, when expressed as CV\%) was slightly higher than that of another study ( $8.3 \%$ in 1 human subject with a sagittal scan 4 minutes and 26 seconds 240 in length (6)). This difference may be because the knee flexion angle used was based on a goniometer measure, not a calculation and that only $30^{\circ}$ and $90^{\circ}$ angles were assessed (6). The spline fitting technique appears to cause the data to diverge at certain points and therefore may overestimate the actual repeatability error.

The differences between MRI contact area centroid and reference standard centroid locations was

245 small, while differences in contact patch overlap were not a consistent overestimation by the MRI-based measures as one might expect due to image resolution. The largest errors in the centroid location results were in the proximal-distal direction; three of the four specimens had MRI-based centroids positioned more proximal than the centroids from the reference standard. Not surprisingly, the contact patch overlap error was in the same direction in the same three specimens. There are several potential reasons a 250 constant overestimation was not observed, including the registration error (up to $0.4 \mathrm{~mm}$ ) between the laser-based surface and the MRI-based surface, the pattern of relaxation in the loaded cartilage (although time between the MRI and cartilage measures was kept to a minimum), and potential alterations in the loading setup (although every attempt was made not to alter it when the MRI bed was retracted from the scanner).

While the results show that the multiplication by slice thickness technique provides the best agreement with the reference standard, the linear and spline interpolation techniques may provide a better description of the actual cartilage surface topology. The difference in error between calculation methods was more apparent when measurements were made from the axial scans (13.8, 40.2 and $37.7 \%$ for the slice thickness multiplication, linear interpolation and spline interpolation, respectively for the delineation method) as compared to the sagittal scans (10.7, 14.5 and $14.7 \%$, in the same order). Theoretically, the multiplication by thickness technique will underestimate the contact area when compared to a linear 
interpolation method. This can be best visualized in two dimensions as the contact area analysis using the adjacent rather than the hypotenuse of the right-angled triangle created between slices. The greater differences in the axial results are likely because there are larger changes in topology in the slice direction 265 and there is less information available to describe the contact area periphery. From the quantitative values of area, the MRI assessment was consistently greater than the reference standard; however, the overestimation was not consistent around the entire periphery. Some of the overestimation of the MRIbased assessment was therefore likely moderated by the underestimation of the multiplication by slice thickness technique. Although, the slice thickness multiplication technique may not provide an adequate 270 description of the surface topology, it appears to be of greater concern in axial assessments.

The patterns of proximal translation and increase in contact area with knee flexion observed in the intra-subject repeatability results are similar to those reported in the literature previously. Both the in vivo and ex vivo contact area migrates proximally with knee flexion $(4,13,17,18,21,23,36,37)$. This was generally the case for the intra-subject repeatability analysis. The variation observed between trials may

275 have been due to measurement errors; however, it is also possible that the patella was positioned differently between trials due to potential differences in muscle recruitment and fatigue. The range of knee flexion angles studied (maximum $35^{\circ}$ and $52^{\circ}$ for Subjects 1 and 2, respectively) was limited by the size of the MRI-scanner's bore and therefore the range of contact area locations was also limited. The results also suggest that Subject 1 has substantial hyperextension of the knee, although this is not 280 necessarily the case since flexion here is described between the anatomical, not the mechanical axes. Correction would be possible if the hip and ankle were scanned also but this is outside the scope of this study and is not of great concern because coordinate systems were defined in the same manner for all participants. The proximally located range of contact areas displayed by Subject 2 was surprising because tendo-femoral contact does not usually occur until $120^{\circ}$ of knee flexion in cadaver studies (23); however, 285 contact area locations are not well characterized in vivo since most studies do not include centroid location. 
The primary strength of this study is that the sagittal MRI scan developed is the fastest scan to be validated to date and the errors of the contact area assessment were not compromised. This scan can be used in series with the scan sequence required for kinematic assessment while still maintaining a total 290 imaging time of one and a half minutes. Other methods that have also assessed kinematics simultaneously require scans of between 4 and 7 minutes (although these methods haven't been well validated) $(6,8,11,13)$ which is a problem because some participants, especially those with patellofemoral disorders (such as patellofemoral pain and osteoarthritis), may not be able to maintain the loaded position for this length of time without substantial artefact. One limitation of the study was the joint capsule was

295 opened. However, since this is a validation study it is not necessary that the contact area be biofidelic just the same between the reference standard and MRI-based assessments. There was a time lapse of approximately 5 minutes between the MRI-based and reference standard assessments and therefore there may be small differences in absolute contact area between assessments (20). Further, once the dye was applied, the cartilage surface was dried and there was an additional time lapse for transportation of the 300 specimen from the MRI centre to the biomechanics laboratory, therefore the contact area digitization and the surface topology data were acquired approximately 1 hour after dye application. There was also error associated with digitizing the periphery of the dye-based contact area. The end of the digitizing probe is a $1 \mathrm{~mm}$ diameter ball bearing which had to be lightly placed on the cartilage surface so as not to compress the cartilage. An attempt was made to minimize this error by using the average of 3 digitization trials.

305 The registration of the digitized area to the laser surface was carried out using the fiducials markers as an initial position estimate. This was done using a single point on the top of each fiducial and may not perfectly align with the point chosen from the laser scanner point cloud. However, the fiducials points were included in the ICP assessment and therefore were allowed to find their optimal position based on the algorithm. A sensitivity analysis showed that variation in choosing these points did not affect the 310 convergence to the correct solution. Finally, intra-subject repeatability was assessed in only two subjects because the imaging took in excess of 3.5 hours to complete. This was therefore quite onerous for the 
participants and fatigue may contribute to differences between trials. Intra-subject repeatability was only assessed in healthy subjects and the applicability of these measures to diseased or injured participants is not clear.

This protocol yields assessments of contact area in less than a minute that have errors similar to those made using scans many times longer. This sequence can be used in series with kinematic scans to assess contact areas and kinematics in vivo using MRI. 


\section{Acknowledgements}

320 We would like to thank Laura Callan and Katharine Wilson for helping with data collection and Trudy Harris, Paul Hammil, Linda James, Burkhart Maedler of the University of British Columbia MRI Centre for their technical assistance. This project was funded by the Canadian Institutes of Health Research (DW- Operating Grant MOP-106680), the Natural Sciences and Engineering Research Council (EM), the

Canadian Arthritis Network (DW, EM), the Michael Smith Foundation for Health Research (EM) and 325 Ireland Canada University Foundation (CO). 


\section{References (max 40)}

1. Salsich GB, Ward SR, Terk MR, Powers CM. In vivo assessment of patellofemoral joint contact area in individuals who are pain free. Clin Orthop Relat Res 2003(417):277-284.

330 2. Heino Brechter J, Powers CM, Terk MR, Ward SR, Lee TQ. Quantification of patellofemoral joint contact area using magnetic resonance imaging. Magn Reson Imaging 2003;21(9):955-959.

3. Besier TF, Draper CE, Gold GE, Beaupre GS, Delp SL. Patellofemoral joint contact area increases with knee flexion and weight-bearing. J Orthop Res 2005;23(2):345-350.

4. Connolly KD, Ronsky JL, Westover LM, Kupper JC, Frayne R. Differences in patellofemoral contact mechanics associated with patellofemoral pain syndrome. J Biomech 2009;42(16):28022807.

5. Gold GE, Besier TF, Draper CE, Asakawa DS, Delp SL, Beaupre GS. Weight-bearing MRI of patellofemoral joint cartilage contact area. J Magn Reson Imaging 2004;20(3):526-530.

6. von Eisenhart-Rothe R, Siebert M, Bringmann C, Vogl T, Englmeier KH, Graichen H. A new in vivo technique for determination of 3D kinematics and contact areas of the patello-femoral and tibio-femoral joint. J Biomech 2004;37(6):927-934.

7. Heino Brechter J, Powers CM. Patellofemoral stress during walking in persons with and without patellofemoral pain. Med Sci Sports Exerc 2002;34(10):1582-1593.

8. Patel VV, Hall K, Ries M, et al. Magnetic resonance imaging of patellofemoral kinematics with weight-bearing. J Bone Joint Surg Am 2003;85-A(12):2419-2424.

9. Nakagawa S, Kadoya Y, Kobayashi A, Tatsumi I, Nishida N, Yamano Y. Kinematics of the patella in deep flexion. Analysis with magnetic resonance imaging. J Bone Joint Surg Am 2003;85-A(7):1238-1242. 
10. Hinterwimmer S, Gotthardt M, von Eisenhart-Rothe R, et al. In vivo contact areas of the knee in patients with patellar subluxation. J Biomech 2005;38(10):2095-2101.

11. Hinterwimmer S, von Eisenhart-Rothe R, Siebert M, Welsch F, Vogl T, Graichen H. Patella kinematics and patello-femoral contact areas in patients with genu varum and mild osteoarthritis. Clin Biomech (Bristol, Avon) 2004;19(7):704-710.

12. Powers CM, Ward SR, Chan LD, Chen YJ, Terk MR. The effect of bracing on patella alignment and patellofemoral joint contact area. Med Sci Sports Exerc 2004;36(7):1226-1232.

13. Shin CS, Carpenter RD, Majumdar S, Ma CB. Three-dimensional in vivo patellofemoral kinematics and contact area of anterior cruciate ligament-deficient and -reconstructed subjects using magnetic resonance imaging. Arthroscopy 2009;25(11):1214-1223.

14. Black JD, Matejczyk MB, Greenwald AS. Reversible cartilage staining technique for defining articular weight-bearing surfaces. Clin Orthop Relat Res 1981(159):265-267.

15. Matthews LS, Sonstegard DA, Henke JA. Load bearing characteristics of the patello-femoral joint. Acta Orthop Scand 1977;48(5):511-516.

16. Yao JQ, Seedhom BB. A new technique for measuring contact areas in human joints--the '3S technique'. Proc Inst Mech Eng H 1991;205(2):69-72.

365 17. Goodfellow J, Hungerford DS, Zindel M. Patello-femoral joint mechanics and pathology. 1. Functional anatomy of the patello-femoral joint. J Bone Joint Surg Br 1976;58(3):287-290.

18. Fujikawa K, Seedhom BB, Wright V. Biomechanics of the patello-femoral joint. Part I: A study of the contact and the congruity of the patello-femoral compartment and movement of the patella. Eng Med 1983;12(1):3-11. 
370 19. Ateshian GA, Kwak SD, Soslowsky LJ, Mow VC. A stereophotogrammetric method for determining in situ contact areas in diarthrodial joints, and a comparison with other methods. $\mathrm{J}$ Biomech 1994;27(1):111-124.

20. Seedhom BB, Tsubuku M. A technique for the study of contact between visco-elastic bodies with special reference to the patello-femoral joint. J Biomech 1977;10(4):253-260.

375 21. Ahmed AM, Burke DL, Yu A. In-vitro measurement of static pressure distribution in synovial joints--Part II: Retropatellar surface. J Biomech Eng 1983;105(3):226-236.

22. Hille E, Schulitz KP, Henrichs C, SchneiderT. Pressure and contract-surface measurements within the femoropatellar joint and their variations following lateral release. Arch Orthop Trauma Surg 1985;104(5):275-282.

380 23. Huberti HH, Hayes WC. Patellofemoral contact pressures. The influence of q-angle and tendofemoral contact. J Bone Joint Surg Am 1984;66(5):715-724.

24. Huberti HH, Hayes WC. Contact pressures in chondromalacia patellae and the effects of capsular reconstructive procedures. J Orthop Res 1988;6(4):499-508.

25. Powers CM, Lilley JC, Lee TQ. The effects of axial and multi-plane loading of the extensor mechanism on the patellofemoral joint. Clin Biomech (Bristol, Avon) 1998;13(8):616-624.

26. Soslowsky LJ, Flatow EL, Bigliani LU, Pawluk RJ, Ateshian GA, Mow VC. Quantitation of in situ contact areas at the glenohumeral joint: a biomechanical study. J Orthop Res 1992;10(4):524534.

27. Goudakos IG, Konig C, Schottle PB, et al. Stair climbing results in more challenging patellofemoral contact mechanics and kinematics than walking at early knee flexion under physiological-like quadriceps loading. J Biomech 2009;42(15):2590-2596. 
28. Luyckx T, Didden K, Vandenneucker H, Labey L, Innocenti B, Bellemans J. Is there a biomechanical explanation for anterior knee pain in patients with patella alta?: influence of patellar height on patellofemoral contact force, contact area and contact pressure. J Bone Joint Surg Br 2009;91(3):344-350.

29. Melegari TM, Parks BG, Matthews LS. Patellofemoral contact area and pressure after medial patellofemoral ligament reconstruction. Am J Sports Med 2008;36(4):747-752.

30. Boehnen C, Flynn P. Accuracy of 3D scanning technologies in a face scanning scenario. Fifth International Conference on 3-D Digital Imaging and Modeling, Proceedings 2005:310-317.

400 31. Fellows RA, Hill NA, Gill HS, et al. Magnetic resonance imaging for in vivo assessment of threedimensional patellar tracking. J Biomech 2005;38(8):1643-1652.

32. Fellows RA, Hill NA, Macintyre NJ, Harrison MM, Ellis RE, Wilson DR. Repeatability of a novel technique for in vivo measurement of three-dimensional patellar tracking using magnetic resonance imaging. J Magn Reson Imaging 2005;22(1):145-153.

405 33. Besl PJ, McKay ND. A method for registration of 3-D shapes. IEEE Transaction on Pattern Analysis and Machine Intelligence 1992;14(2):239-255.

34. McWalter EJ, Hunter DJ, Wilson DR. The effect of load magnitude on three-dimensional patellar kinematics in vivo. J Biomech 2010;43(10):1890-1897.

35. McWalter EJ, Hunter DJ, Harvey WF, et al. The effect of a patellar brace on three-dimensional patellar kinematics in patients with lateral patellofemoral osteoarthritis. Osteoarthritis Cartilage 2011;19(7):801-808.

36. Kwak SD, Ahmad CS, Gardner TR, et al. Hamstrings and iliotibial band forces affect knee kinematics and contact pattern. J Orthop Res 2000;18(1):101-108. 
37. Hefzy MS, Jackson WT, Saddemi SR, Hsieh YF. Effects of tibial rotations on patellar tracking and patello-femoral contact areas. J Biomed Eng 1992;14(4):329-343. 


\section{Tables}

Table I: T1-weighted MRI sequence parameters.

\begin{tabular}{lccccc}
\hline Parameter & $\begin{array}{c}\text { Unloaded Bone } \\
\text { Sagittal }\end{array}$ & $\begin{array}{c}\text { Loaded Bone } \\
\text { Sagittal }\end{array}$ & $\begin{array}{c}\text { Unloaded } \\
\text { Cartilage } \\
\text { Sagittal }\end{array}$ & $\begin{array}{c}\text { Loaded } \\
\text { Cartilage } \\
\text { Sagittal }\end{array}$ & $\begin{array}{c}\text { Loaded } \\
\text { Cartilage Axial }\end{array}$ \\
\hline Scan Sequence & MS SE & MS SE & 3 DFF & 3 D FFE & 3 DFFE \\
In-plane Resolution* & $0.60 \mathrm{~mm}$ & $1.25 \mathrm{~mm}$ & $0.60 \mathrm{~mm}$ & $0.3125 \mathrm{~mm}^{\star}$ & $0.3125 \mathrm{~mm}^{\star}$ \\
Field of View & $300 \mathrm{~mm}$ & $320 \mathrm{~mm}$ & $300 \mathrm{~mm}$ & $180 \mathrm{~mm}$ & $180 \mathrm{~mm}$ \\
Slice Thickness & $2 \mathrm{~mm}$ & $2 \mathrm{~mm}$ & $2 \mathrm{~mm}$ & $2 \mathrm{~mm}$ & $2 \mathrm{~mm}$ \\
Slice Separation & $2 \mathrm{~mm}$ & $7 \mathrm{~mm}$ & $2 \mathrm{~mm}$ & $2 \mathrm{~mm}$ & $2 \mathrm{~mm}$ \\
Matrix Size & $512 \times 512$ & $256 \times 256$ & $512 \times 512$ & $576 \times 576$ & $576 \times 576$ \\
Repetition Time & $360 \mathrm{~ms}$ & $306 \mathrm{~ms}$ & $12 \mathrm{~ms}$ & $11 \mathrm{~ms}$ & $14 \mathrm{~ms}$ \\
Echo Time & $10.0 \mathrm{~ms}$ & $6.2 \mathrm{~ms}$ & $4.3 \mathrm{~ms}$ & $3.9 \mathrm{~ms}$ & $4.6 \mathrm{~ms}$ \\
Flip Angle & $90^{\circ}$ & $90^{\circ}$ & $15^{\circ}$ & $10^{\circ}$ & $10^{\circ}$ \\
Scan Time & $7 \mathrm{~min} 44 \mathrm{~s}$ & $35 \mathrm{~s}$ & $4 \mathrm{~min} 53 \mathrm{~s}$ & $58 \mathrm{~s}$ & $52 \mathrm{~s}$ \\
MRI Coil & Knee & Body & Knee & Body & Body
\end{tabular}

*Indicates the reconstructed in plane resolution, the loaded cartilage scans were acquired at $0.4 \times 0.4 \mathrm{~mm}$.

$M S=$ multi-shot, $S E=$ spin echo, $3 D=$ three-dimensional, $F F E=$ fast field echo 
425 Table II: Error of the MRI-based assessment of contact area as compared to the reference standard.

\begin{tabular}{lcccc}
\hline & \multicolumn{2}{c}{ Sagittal } & \multicolumn{2}{c}{ Axial } \\
& Delineation & Intersection & Delineated & Intersection \\
\hline Multiply by slice & $47.7 \pm 38.1$ & $59.4 \pm 29.6$ & $61.4 \pm 75.2$ & $60.5 \pm 57.2$ \\
thickness & $(10.7 \%)$ & $(13.9 \%)$ & $(13.8 \%)$ & $(13.7 \%)$ \\
Linear Interpolation & $63.5 \pm 48.9$ & $68.4 \pm 46.4$ & $173.0 \pm 124.3$ & $175.6 \pm 120.6$ \\
& $(14.5 \%)$ & $(15.6 \%)$ & $(40.2 \%)$ & $(40.8 \%)$ \\
Spline Interpolation & $64.1 \pm 48.8$ & $67.5 \pm 44.7$ & $162.7 \pm 124.7$ & $170.1 \pm 122.9$ \\
& $(14.7 \%)$ & $(15.4 \%)$ & $(37.7 \%)$ & $(39.4 \%)$ \\
\hline
\end{tabular}

Error is expressed as mean absolute difference \pm standard deviation $\left(\mathrm{mm}^{2}\right)$ and percentage difference (in brackets). 
Table III: Intra-reader repeatability.

\begin{tabular}{lcccc}
\hline & \multicolumn{2}{c}{ Sagittal } & \multicolumn{2}{c}{ Axial } \\
& Delineation & Intersection & Delineated & Intersection \\
\hline Multiply by slice thickness & $9.4 \pm 4.3$ & $21.7 \pm 7.7$ & $8.4 \pm 4.1$ & $20.8 \pm 13.1$ \\
Linear Interpolation & $9.1 \pm 2.5$ & $22.9 \pm 7.1$ & $15.4 \pm 14.1$ & $25.9 \pm 24.1$ \\
Spline Interpolation & $9.3 \pm 2.0$ & $22.0 \pm 7.1$ & $15.0 \pm 14.6$ & $26.2 \pm 24.1$ \\
\hline
\end{tabular}

430 Expressed as the mean standard deviation and the mean standard deviation \pm standard deviation $\left(\mathrm{mm}^{2}\right)$ 


\section{Figure Legends}

Figure 1: Schematic of cadaver knee specimen in MRI-safe loading apparatus positioned in the MRI scanner.

Figure 2: Example of axial (top) and sagittal (bottom) scans delineated (left) and segmented (right).

Figure 3: Centroids of the MRI and three trials of the reference standard contact areas. The blue lines represent the 3 reference standard trials while the blue marker represents the mean reference standard centroid. The red line and marker represent the MRI based assessment of contact using the sagittal scan and the delineation method. $\mathrm{M}=$ medial, $\mathrm{L}=$ lateral.

Figure 4: Contact area intra-subject repeatability results presented for three trials for two subjects. Repeatability was assessed over the coincidental range of the three trials only. Raw data are denoted by 445 markers, splines are denoted by lines. This graph uses the spline interpolation area calculation technique; however, results were similar for all techniques.

Figure 5: Contact area contours and centroids for three trials in two subjects. Contours and centroids were colour coded with respect to the knee flexion angle at which they were assessed, which differed between trials 450 because of the inherent error in the goniometer used for positioning and the use of the calculated flexion angle in the analysis. 


\section{$455 \quad$ Figures}

Figure 1:

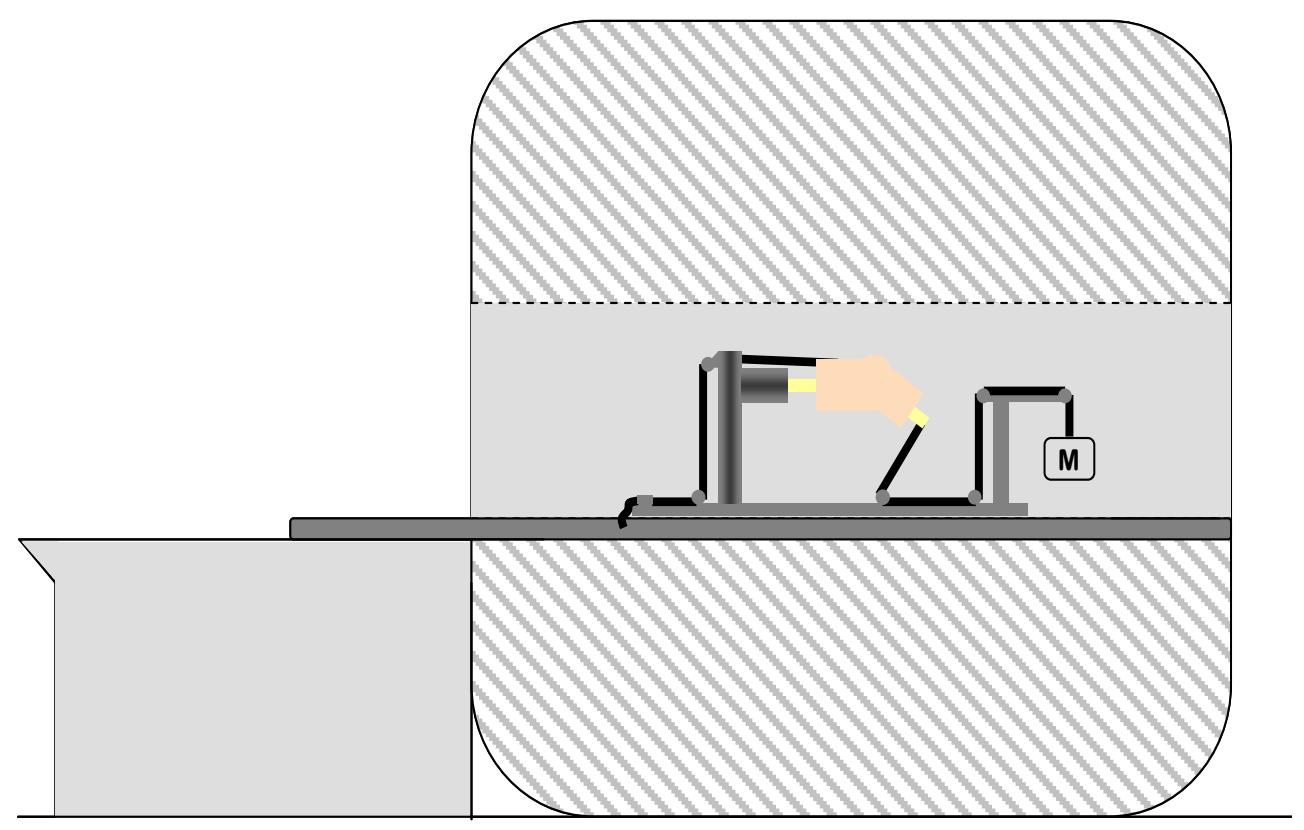


Figure 2
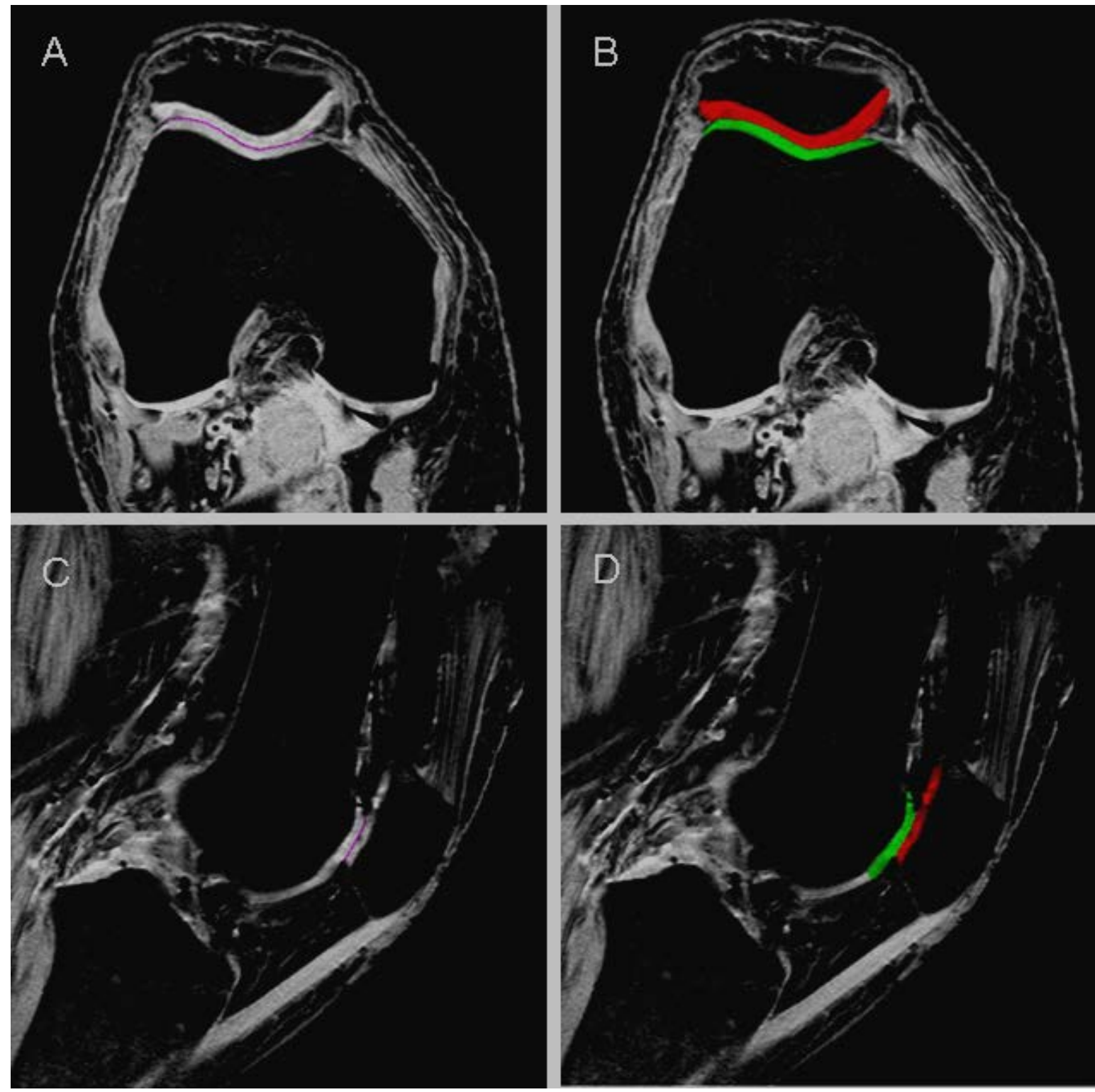

460 
Figure 3:

Specimen 1

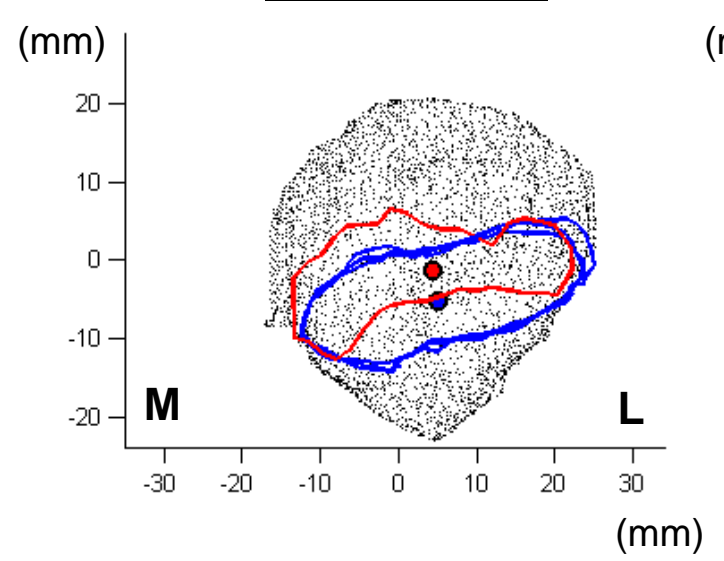

Specimen 2

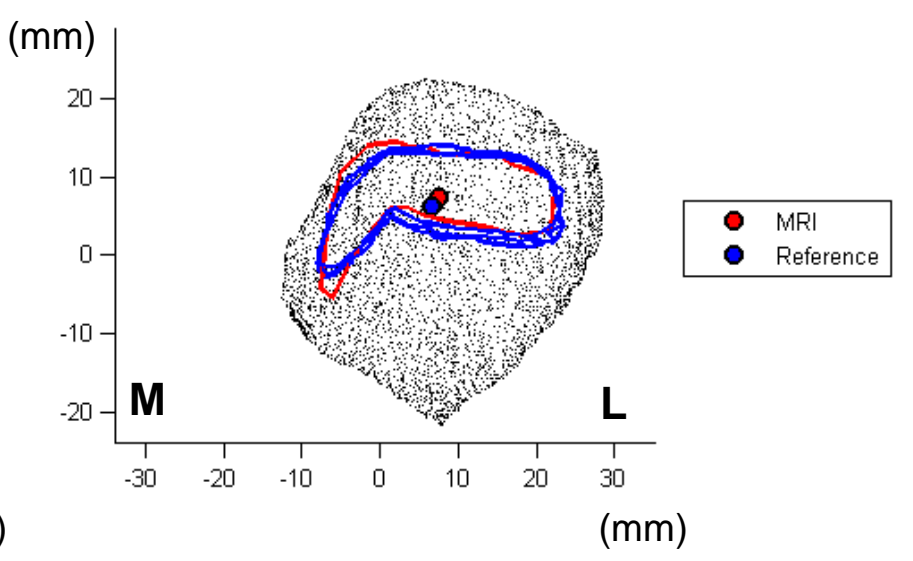

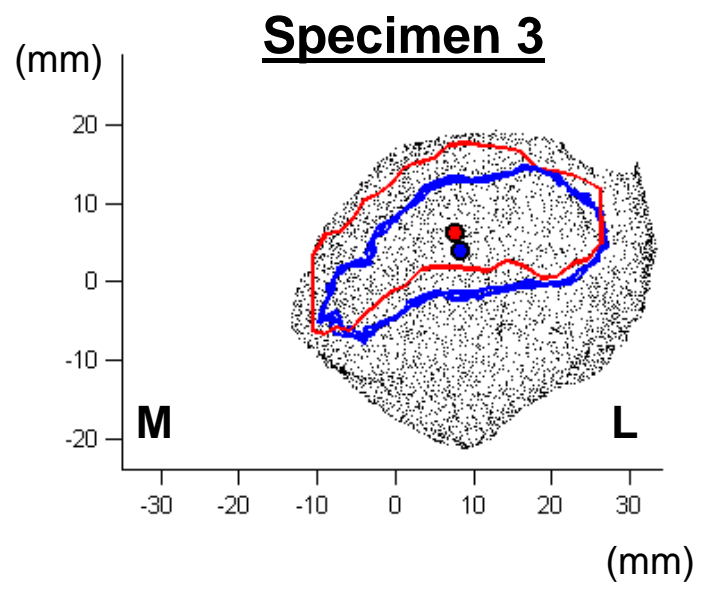

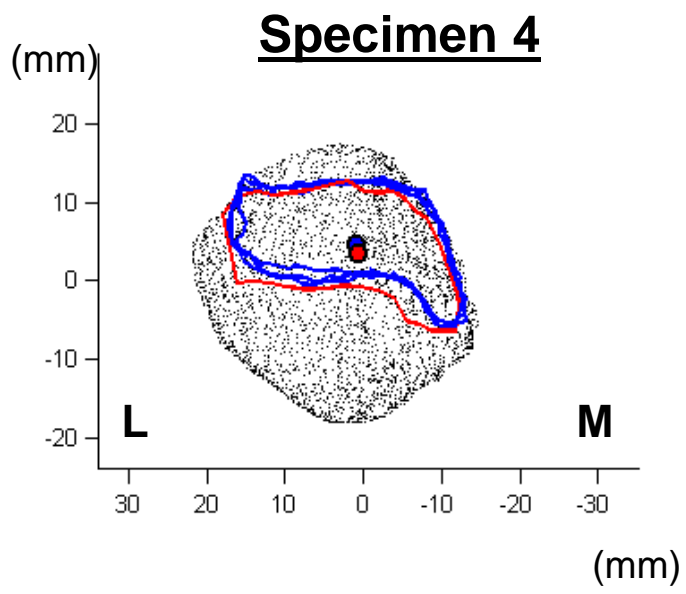

465 
Figure 4:

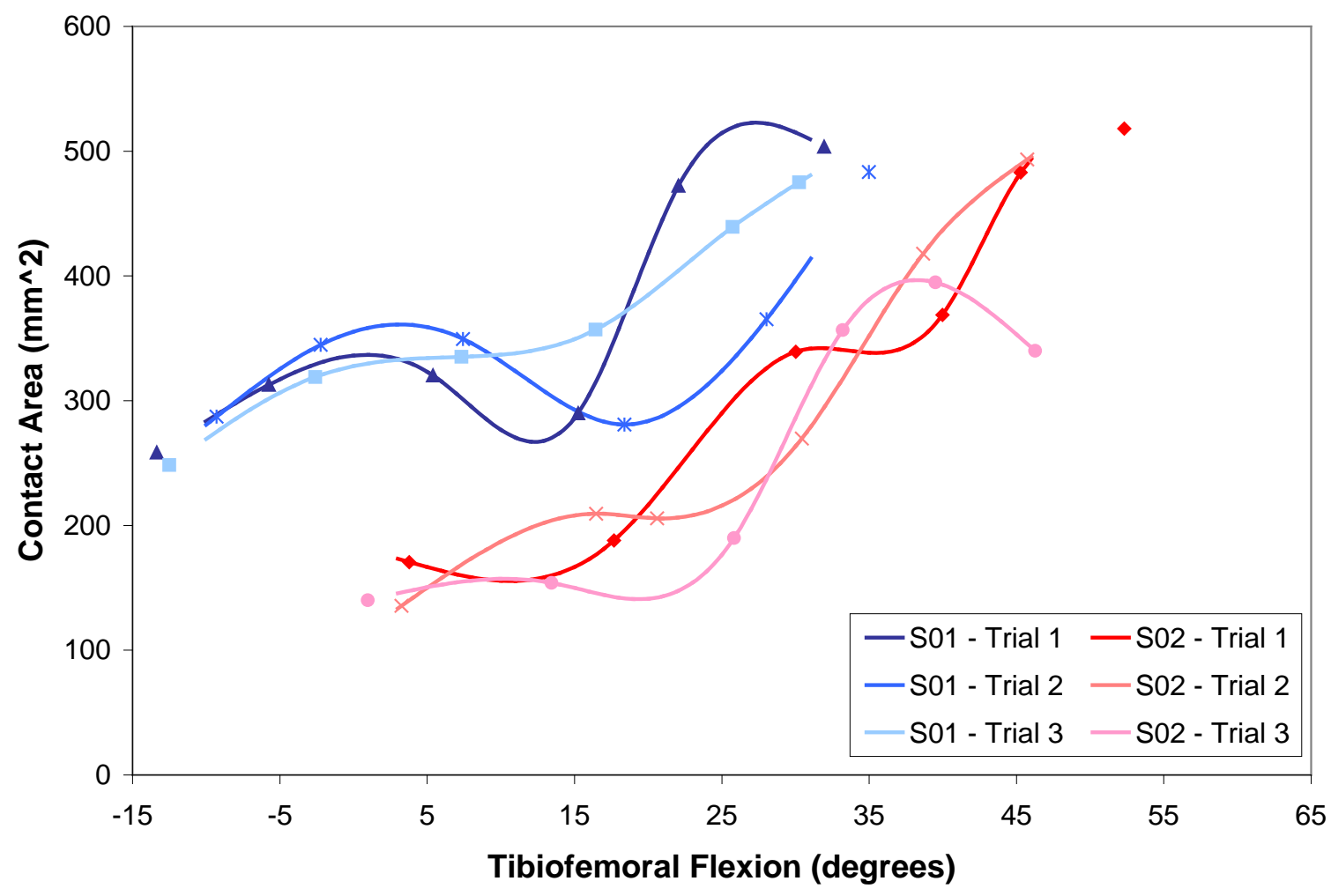


Figure 5:

Subject 1
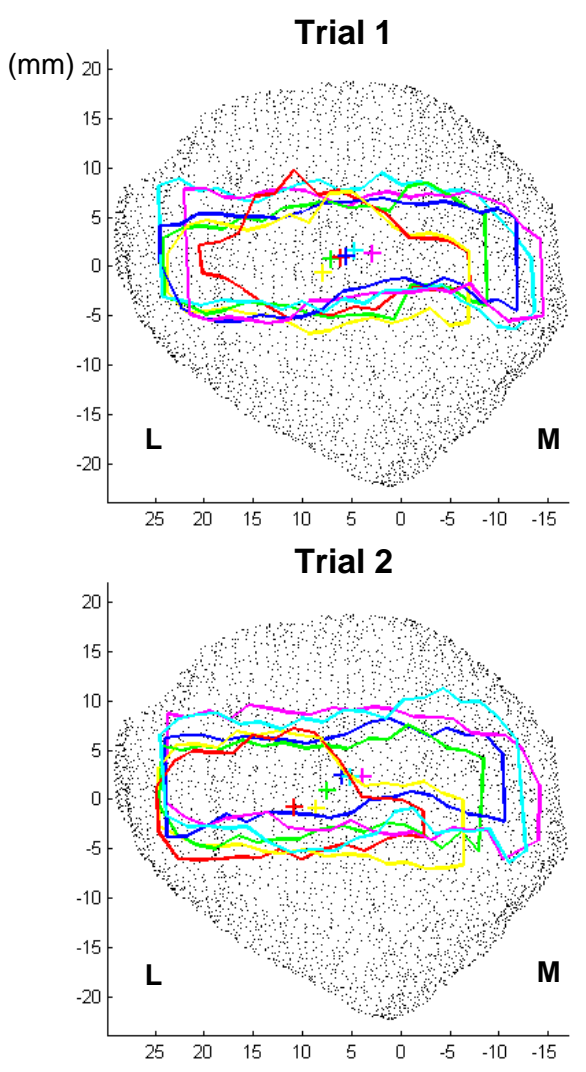

Trial 3

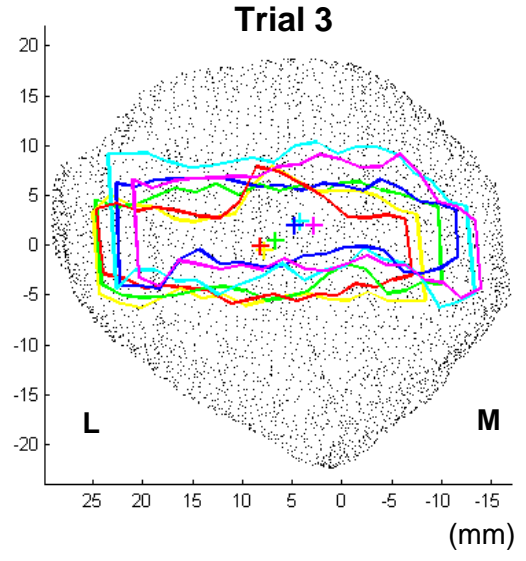

\section{Subject 2}

Trial 1

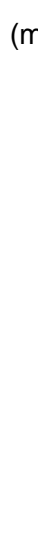

(mm) 20
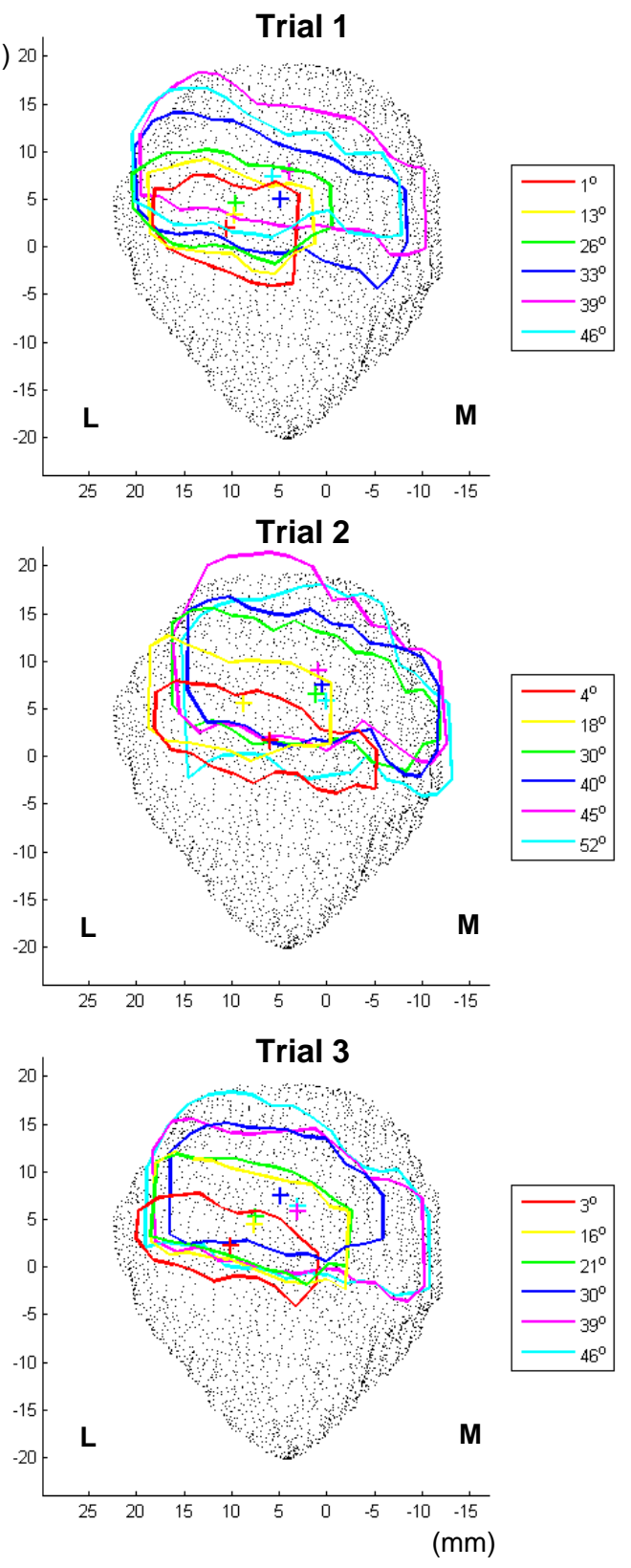
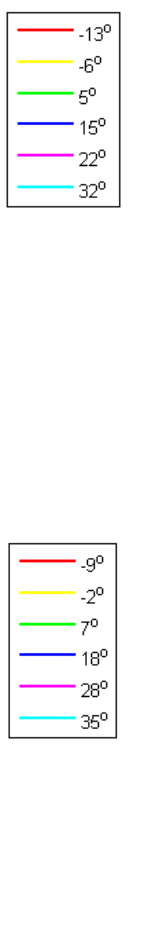